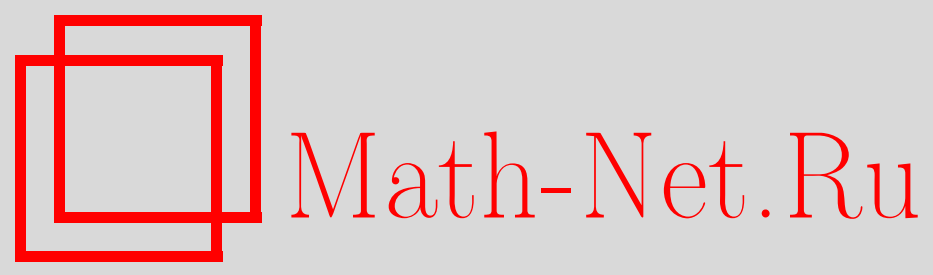

А. Р. Чехлов, Об одном классе эндотранзитивных групп, Матем. заметки, 2001, том 69, выпуск 6, 944-949

DOI: https://doi.org/10.4213/mzm709

Использование Общероссийского математического портала Math-Net.Ru подразумевает, что вы прочитали и согласны с пользовательским соглашением http://www.mathnet.ru/rus/agreement

Параметры загрузки:

IP: 54.92 .164 .108

26 апреля 2023 г., 04:09:13

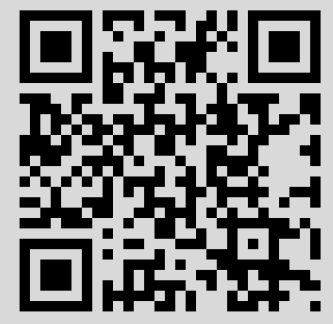




\title{
ОБ ОДНОМ КЛАССЕ ЭНДОТРАНЗИТИВНЫХ ГРУПП
}

\author{
А.Р. Чехлов
}

Получено описание редуцированных эндотранзитивных групп без кручения со свойством максимальности на ядра эндоморфизмов, тип любого ненулевого элемента которых сравним с некоторьм максимальньм типом в множестве типов всех ненулевых элементов группы.

Библиографоия: 11 названий.

Абелева группа без кручения $A$ называется әндотранзитивной (транзитивной), если для любых $0 \neq a, b \in A$ условие на их характеристики $\chi(a)=\chi(b)$ влечет существование эндоморфизма (автоморфизма) $f$ этой группы со свойством $f a=b$. Если такой эндоморфизм $f$ найдется при $\chi(a) \leqslant \chi(b)$ для любых $0 \neq a, b \in A$, то $A$ назьвается вполне транзитивной. Транзитивные и вполне транзитивныегрупшы без кручения изучались в работах [1]-[9] и др., см. также обзоры [10], [11, с. 367]. Всякая транзитивная и вполне транзитивная группа без кручения являются эндотранзитивными. Поэтому изучение эндотранзитивных групп представляет интерес, поскольку оно позволяет выработать определенньй подход к исследованию транзитивных, вполне транзитивных и других, близких к ним классов групп. Эндотранзитивные групшы без кручения исследовались в $[1],[2]$. Однородные эндотранзитивные групшы под названием $E$-транзитивные изучались и в [7], [8], они совпадают с классом однородных вполне транзитивных групп. Нередуцированная группа без кручения эндотранзитивна тогда и только тогда, когда ее редуцированная часть эндотранзитивна. Прямое слагаемое эндотранзитивной группы эндотранзитивно. Отметим, что свойство эндотранзитивности грушшы без кручения $A$ эквивалентно такому свойству: для любых $0 \neq a, b \in A$ таких, что $t(a)=t(b)$ и $\chi(a) \leqslant \chi(b)$, существует ее эндоморфизм, переводящий $a$ в $b[1$, предложение 1.1$]$.

Обозначим через $\mathscr{L}$ класс всех редуцированных групп без кручения $A$ таких, что для любого $0 \neq a \in A$ тип $t(a)$ сравним с некоторым максимальным типом в множестве $T(A)$ типов всех ненулевых элементов группы $A$. В этой статье получено описание эндотранзитивных групा из $\mathscr{L}$, ядра эндоморфизмов которых обладают свойством максимальности. Таковы, например, все групшы без кручения конечного ранга. Поэтому данные результаты обобщают результаты $\S 4$ из [1], описьвающие эндотранзитивные группы без кручения конечного ранга. К рассматриваемому классу относятся также квазиоднородные групшы $A$ из $\mathscr{L}$ конечного $p$-ранга хотя бы для одного простого числа $p$ со свойством $p A \neq A$; ранг таких групп может достигать мощности континуума. 
Если $A$ - группа без кручения, то $h_{p}^{A}(a)-p$-высота ее элемента $a$; если $G \subseteq A$, то $\langle G\rangle_{*}^{A}$ - сервантная подгруппа в $A$, порожденная $G$ (индекс $A$ иногда опускаем); $A(t)=$ $\{a \in A \mid t(a) \geqslant t\}$, аналогично определяется подгруппа $A(\chi) ; \Pi(A)$ - множество всех простых чисел $p$ со свойством $p A \neq A ; R$ - кольцо эндоморфизмов группы $A$, т.е. $E(A)$; если $p$ - простое число, то $p$-рангом $A$ называется ранг ее факторгруппы $A / p A ; 1_{A}-$ тождественный эндоморфизм группы $A ; A$ называется квазиоднородной, если $\Pi(G)=$ $\Pi(A)$ для любой ее ненулевой сервантной подгрупшы $G$; квазиразложимой, если существуют ее сервантные подгрупшы $B, G \neq 0$ такие, что $n A \subseteq B \oplus G$ для некоторого натурального числа $n, B$ и $G$ назьваются ее квазислагаемыми. Будем говорить, что $A$ квазиизоморфна группе $B$, если $A \cong G$, где $G$ - такая подгруппа в $B$, что $n B \subseteq G$ для некоторого натурального $n$. Обозначим через $\mathscr{N}$ класс всех редуцированных групп без кручения, все ненулевые эндоморфизмы которых - мономорфизмы. Если $f: A \rightarrow B-$ гомоморфизм, то $f \mid G$ - ограничение $f$ на $G \subseteq A$.

Для замкнутости изложения докажем следующую лемму из [1].

Лемма 1. Пусть $A-$-редуиированная группа без кручения. Тогда

1) если $f \in R, 0 \neq b \in \operatorname{Ker} f u h_{p}(b) \geqslant h_{p}(f a)$, mo $h_{p}(a+b)=h_{p}(a)$; в частнос$m u$, ecли $\chi(b) \geqslant \chi(f a)$, mo $\chi(a+b)=\chi(a) ;$

2) если $A$ әндотранзитивна и подмножество $T \subseteq T(A)$ таково, что для всякого $t \in T(A)$ существует $\tau_{t} \in T$ со свойством $t \leqslant \tau_{t}$, то әндоморфизм $f \in R$, аннулируюший множество $V=\bigcup_{\tau \in T} A(\tau)$, равен нулю.

ДокАЗАТЕЛЬСтво. 1) Имеем $h_{p}(a+b) \geqslant h_{p}(a) \cap h_{p}(b)=h_{p}(a)$. Далее

$$
h_{p}(a+b) \leqslant h_{p}(f(a+b))=h_{p}(f a) \leqslant h_{p}(b),
$$

поэтому

$$
h_{p}(a)=h_{p}((a+b)-b) \geqslant h_{p}(a+b) \cap h_{p}(b)=h_{p}(a+b) .
$$

Следовательно, $h_{p}(a)=h_{p}(a+b)$.

2) Предположим, что $f a \neq 0$. По условию найдется $0 \neq b \in A$ со свойством $\chi(b) \geqslant$ $\chi(f a)$. Пусть $h_{p}(b)<\infty$. Поделив, если нужно, $b$ на соответствующую степень $p$, можно считать, что $h_{p}(b)=h_{p}(a)$. Тогда в силу 1$)$ вьполнено равенство $\chi\left(p^{n} a+b\right)=\chi(a)$, где $n>h_{p}(f a)$. Имеем $p^{n} \varphi a+\varphi b=a$ для некоторого $\varphi \in R$ и $\varphi b \in V$. Откуда $p^{n} f \varphi=f a$. Противоречие.

Лемма 2. Если $A \in \mathscr{L}$ - квазиоднородная әндотранзитивная группа $и A(t) \subseteq$ Ker $f$ для некоторого $t \in T(A)$, mо $f=0$.

ДокАЗАТЕЛЬСтво. Можно считать, что $t=t(a)-$ максимальный тип, пусть $b \in$ $A \backslash p A$ - элемент другого максимального типа. Поделив $a$, если нужно, на соответствующую степень $p$, можно считать, что $a \notin p A$. Покажем, что $t\left(p^{n} b+a\right)=t(b+a)$ для любого натурального $n$. Предположим, что $q^{k} x=p^{n} b+a$, где $q$-простое число $\neq p, k-$ натуральное. Тогда $q^{k} f x=p^{n} f b$. Так как $\chi(f b)=\chi(s b)$ для некоторого натурального $s$, то $q^{k} \mid s b$. Если $(q, s)=1$, то $q^{k} \mid b$ и, значит, $q^{k} \mid(b+a)$. Итак, $t\left(p^{n} b+a\right) \leqslant t(b+a)$. Аналогично, $t\left(p^{n} b+a\right) \geqslant t(b+a)$. Следовательно, найдутся взаимно простые натуральные $m$ и $r$, не делящиеся на $p$, со свойством $\chi\left(p^{n} m b+m a\right)=\chi(r b+r a)$. Тогда 
$p^{n} f b+f a=r b+r a$. В силу произвольности $n$ из этого следует, что $r b$ и, значит, $b$ принадлежат замыканию в $p$-адической топологии группы $A$ ее подгруппы $A(t)$. Поскольку $f A(t)=0$, то $f b$ принадлежит $p$-делимой подгруппе группы $A$. В силу квазиоднородности $f b=0$. Но тогда по лемме $1 f=0$.

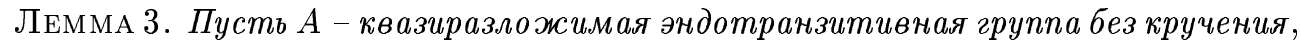
$m A \subseteq B \oplus G$, где $0 \neq B, G-$ сервантные подгруппь в $A$. Тогда

1) если $0 \neq f: G \rightarrow B$ - гомоморфизм, то найдется гомоморфизм $\eta: B \rightarrow G$ со свойством $\eta f \neq 0 ;$ в частности, если $G \in \mathcal{N}$, то все ненулевые гомоморфизмы $\varphi: G \rightarrow A-$ мономорфизмы;

2) если $G \in \mathscr{N}$, то $G$ - квазиоднородная эндотранзитивная группа;

3) если $0 \neq a \in A$, то при условии $\Pi(B) \cap \Pi\left(\langle a\rangle_{*}\right) \neq \varnothing$ найдется $\tau \in T(B)$ со свойством $\tau \geqslant t(a)$

4) если $0 \neq a \in A$ - әлемент максимального типа $t$ u $t \in T(B), T(G)$, то $t(z) \leqslant t$ для любого $z \in A$ с условием $\Pi\left(\langle z\rangle_{*}\right) \cap \Pi\left(\langle a\rangle_{*}\right) \neq \varnothing ;$ в частности, $t$ - наибольший тип в $T(A)$, если $A$ квазиоднородна;

5) если $t \in T(G)$ - максимальный тип, то $t$ максимален в $T(A)$; в этом случае условие $G \in \mathscr{N}$ влечет, что $B$ - прямое слагаемое в $A$ с дополнительным прямым слагаемым $D \cong G$, причем $D$ - однородная группа при $\Pi(B) \cap \Pi(G) \neq \varnothing$.

ДокАЗАтЕльство. 1) Докажем более общий факт. Если $t(b) \geqslant t(g)$ для некоторых $0 \neq b \in B, g \in G$, то найдется $\eta: B \rightarrow G$ со свойством $\eta b \neq 0$. Можно считать, что $\chi(b) \geqslant \chi(g)$, и пусть $p$ - такое простое число, что $h_{p}(b)<\infty$. Как и в лемме 2 , можно считать, что $h_{p}(b)=h_{p}(g)=0$. Тогда $\chi\left(b+p^{n} g\right) \leqslant \chi(m g)$, причем типы этих элементов совпадают, так как $b, g$ из взаимно дополнительных квазислагаемых, где натуральное $n$ таково, что $p^{n}$ не делит $m^{2}$. Поэтому $f b+p^{n} f g=m g$ для некоторого $f \in R$. Если эндоморфизм $\pi \in R$ такой, что $\pi \mid G=m \cdot 1_{G}$ и $\operatorname{Ker} \pi=B$, то $\pi f b+p^{n} \pi f g=m^{2} g$. Имеем $\pi f: B \rightarrow G$ и $\pi f b \neq 0$ в силу выбора $n$. Пусть теперь $G \in \mathcal{N}$. Если $f: G \rightarrow A$ и $\operatorname{Ker} f \neq 0$, то $f G \nsubseteq G$. Тогда если эндоморфизм $\theta \in R$ такой, что $\theta G=0$ и $\theta \mid B=m \cdot 1_{B}$, то $\theta f \neq 0$. По доказанному найдется $\eta: B \rightarrow G$ со свойством $\eta \theta f \neq 0$, это эндоморфизм группы $G$ с ненулевым ядром.

2) Если $a, b \in G$ и $\chi(a)=\chi(b)$, то $f a=b$ для некоторого $f \in R$. Имеем $f \mid G \in E(G)$. В противном случае $0 \neq \theta f: G \rightarrow A$ и $\operatorname{Ker} \theta f \neq 0$, где $\theta$ - такой же эндоморфизм, как в 1$)$. Итак, $G$ эндотранзитивна. Если $h_{p}(g)=\infty$, где $0 \neq g \in G$, то $\chi(p g)=\chi(g)$. Поэтому $p f g=g$ для некоторого $f \in R$ и, значит, $1_{G}=p f$. Откуда следует, что $p G=G$. Это доказьвает ее квазиоднородность.

3) Имеем $m a=b+g$, где $b \in B, g \in G, t(a)=t(m a) \leqslant t(b), t(g)$. Если $b \neq 0$, то $\tau=t(b)$. Предположим, что $b=0$. Тогда $a \in G$. По условию найдется $b \in B \backslash p B$, где $p$ - такое простое число, что $h_{p}(a)<\infty$. Как и вьше, считаем, что $h_{p}(a)=0$. Имеем $\chi\left(a+p^{n} b\right) \leqslant \chi(m a+m b)$, где типы этих элементов совпадают и $n$ такое же, как в 1$)$. Поэтому, аналогично 1), $\theta f a+p^{n} \theta f b=m^{2} b$. Здесь $0 \neq \theta f a \in B$ и $t(\theta f a) \geqslant t(a)$. Заметим еше, что $t(b) \leqslant t\left(\left(p^{n} \theta f-m^{2} \cdot 1_{B}\right) b\right)=t(\theta f a)$, т.е. если $\Pi\left(\langle b\rangle_{*}\right) \cap \Pi\left(\langle g\rangle_{*}\right) \neq \varnothing$, где $0 \neq b \in B, 0 \neq g \in G$, то существует эндоморфизм группы $B$, переводящий $b$ в некоторый элемент этой группы типа $\geqslant t(g)$. 
4) Пусть $m z=x+y$, где $x \in B, y \in G$. Имеем $t(z) \leqslant t(x), t(y)$. Если $h_{p}(z), h_{p}(a)<\infty$, то, например, $h_{p}(x)<\infty$. По условию существует $0 \neq g \in G$ типа $t$. Поэтому это утверждение вытекает из максимальности $t$ и замечания в конце доказательства 3$)$.

5) Допустим, что нашелся $0 \neq a \in A$ со свойством $t(a)>t=t(g)$, где $g \in G$. Тогда $\Pi(G) \cap \Pi\left(\langle a\rangle_{*}\right) \neq \varnothing$, но это противоречит максимальности $t$ по доказанному свойству 3$)$. Пусть теперь $G \in \mathcal{N}$. Если $\Pi(G) \cap \Pi(B)=\varnothing$, то $B \oplus G$ - сервантная подгруппа в $A$. Действительно, если $p x=b+g$, где $p \in \Pi(A), b \in B, g \in G$, и, например, $p \in \Pi(G)$, то $p B=B$ и $b \in p B$. Откуда $g \in p G$ и, значит, $x \in B \oplus G$. Сервантность влечет совпадение этой подгруппы с $A$.

Предположим, что $\Pi(B) \cap \Pi(G) \neq \varnothing$. Так как по 2) $G$ квазиоднородна, то $\Pi(G)=$ $\Pi\left(\langle g\rangle_{*}\right)$. Тогдапо 3$) t \in T(B)$ и $t$-наибольший тип в $T(G)$ по 4$)$. Если $0 \neq \gamma \in S=E(G)$, то $t(\gamma g)=t(g)$. Поэтому $\chi(\gamma g)=\chi(n g)$ для некоторого натурального $n$ и, значит, $f \gamma g=n g$, где $f \in S$. Откуда $f \gamma=n \cdot 1_{G}$. Следовательно, любой элемент кольца $S$ является целым кратным обратимого. В частности, все ненулевые эндоморфизмы группы $G$ сохраняют типы ее элементов и все левые идеалы кольца $S$ имеют вид $n S$, где $n$ - целые числа. С другой стороны, если $t(x)<t$ для некоторого $x \in G$, то согласно сделанному замечанию в конще доказательства 3 ) существует эндоморфизм группы $G$, переводящий $x$ в некоторьй элемент типа $t$. Это доказьвает однородность $G$. Так как $G$ эндотранзитивна, то отображение $S \ni \gamma \rightarrow \gamma g$ изоморфно вкладывает $S^{+}{ }_{\text {в }}$ $F=G(\chi)$, где $\chi=\chi(g)$. Поскольку для $x, y \in F$ условие $\chi_{F}(x) \leqslant \chi_{F}(y)$ эквивалентно $\chi_{G}(x) \leqslant \chi_{G}(y)$, то любой эндоморфизм групшы $F$ однозначно продолжается до эндоморфизма $G=\langle F\rangle_{*}$. Умножение группы $S^{+}$слева и справа на $0 \neq s \in S$ однозначно действуют на $1 \in S^{+}$. Рассматривая их как эндоморфизмы $S^{+}$, получаем, что $s \gamma=\gamma s$ для любого $\gamma \in S$, т.е. $S$ - коммутативное кольцо главных идеалов. Подгруппу $A(\chi)$ можно рассматривать как $S$-модуль. Действительно, если $b \in A(\chi)$, то $t(b)=t$, так как $t$ - максимальный тип, поэтому $b=\gamma g$ для некоторого $\gamma \in R$. Полагаем $s b=\gamma s g$. Фактормодуль $E=A(\chi) / B(\chi)$ квазиизоморфен циклическому $S$-модулю $G(\chi) \cong S g$. Так как $S$ - кольцо главных идеалов, то $E \cong S^{+}$и, значит, $B(\chi)$ - прямое слагаемое в $A(\chi)=B(\chi) \oplus M$. Имеем $A(t)=\langle B(\chi)\rangle_{*} \oplus D$, где $D=\langle M\rangle_{*}$. Так как $G \subseteq A(t)$, то $A=D \oplus B$. Поскольку $D$ квазиизоморфна $G$, то $D \in \mathscr{N}$.

Теорема 4. Пусть $A$ - эндотранзитивная группа без кручения, $t \in T(A)-$ максимальный тип, әндоморфизм $\alpha \in R$ смаксимальным ненулевым ядром $u$ $\alpha A(t) \neq 0$. Тогда $B=\operatorname{Ker} \alpha-$ прямое слагаемое в $A$ с дополнительным прямым слагаемым $D \in \mathscr{N}$.

ДокАЗАТЕЛЬСтво. Пусть $g \in A(t)$ и $\alpha g \neq 0$. Так как $t(\alpha g)=t(g)$, то $\chi(m g)=\chi(\alpha g)$ для некоторого натурального $m$. Откуда следует, что существует $f \in R$ со свойством $f g=m g$ и $\operatorname{Ker} f=B(\beta \alpha g=m g$ для некоторого $\beta$, тогда $f=\beta \alpha)$. Имеем $\langle B, g\rangle \subseteq \operatorname{Ker}\left(f^{2}-m f\right)$. Значит, $f^{2}=m f$. Откуда $m A \subseteq B \oplus G$, где $G=\langle f A\rangle_{*}$. В силу максимальности $\operatorname{Ker} f$ все ненулевые гомоморфизмы $G \rightarrow A$ являются мономорфизмами. В частности, $G \in \mathcal{N}$. Теперь утверждение теоремы вытекает из леммы 3 , п. 5).

СлЕДСТВИЕ 5. Пусть $A-$ - квазиоднородная группа, удовлетворяющая посылке теоремы 4. Тогда $D$ - однородная әндотранзитивная группа, подгруппа $A(t)$ 
плотна в $A$ в ее $Z$-адической топологии, причем любой әлемент из $A(t)$ можно вложить в прямое слагаемое группы $A$, изоморфное $D$.

ДокАЗАТЕЛЬСТво. Однородность $D$ следует из леммы 3, п. 5 ) в силу квазиоднородности $A$. Имеем $A=B \oplus D$. По лемме 3, п. 4$) t$-наибольший тип в $T(A)$. Если $b \in B \backslash p B$, то $t\left(p^{n} b+x\right)=t(b)$ для любого $x \in D$. Теперь так же, как в доказательстве леммы 2 , $A(t)$ плотна в $A$ в ее $p$-адической топологии для каждого $p \in \Pi(A)$, значит, $A(t)$ плотна в $Z$-адической топологии. Если теперь $0 \neq a \in A(t)$, то найдется $x \in D$ со свойством $\chi(a)=\chi(x)$. Поэтому $f x=a$ и $\varphi a=x$ для некоторых $f, \varphi \in R$. Если $\pi-$ проекция $A$ на $D$, то для $G=f D$ имеем $\delta A=G$ и $\delta \mid G=1_{G}($ так как $G=f D \cong D$ и $\delta a=a)$, т.е. $G-$ прямое слагаемое в $A$.

СледСтВИЕ 6. Пусть $A \in \mathscr{L}$ - квазиоднородная группа, удовлетворяющая условию максимальности на ядра әндоморфизмов (в частности, пусть $A$ имеет конечный р-ранг для некоторого $p \in \Pi(A)), u A \notin \mathscr{N}$. Группа $A$ әндотранзитивна тогда и только тогда, когда она является конечной прямой суммой изоморфных однородных вполне транзитивных групп из класса $\mathcal{N}$.

ДокАЗАТЕЛЬСТво. Необходимость вытекает из леммы 2, следствия 5 и леммы 3 , пп. 3), 4), так как если $A=B \oplus D$, где $D \in \mathcal{N}$, то $B$ - эндотранзитивная группа, удовлетворяющая посылке следствия как прямое слагаемое. Это следует из того, что если $\theta$ - проекция $A$ на $B$, то $f \theta \in R$ для любого $f \in E(B)$. Поэтому $B$ также имеет прямое слагаемое из $\mathcal{N}$. В силу условия на ядра эндоморфизмов разложение группы $A$ в прямую сумму групп должно быть конечным. Достаточность следует из того факта, что конечная прямая сумма однородных вполне транзитивных групп является эндотранзитивной группой [1, теорема 3.1$]$.

СлЕДСТВИЕ 7. Пусть $A \in \mathscr{L}$ - әруппа, удовлетворяющая условию максимальности на ядра эндоморфизмов, $и A \notin \mathscr{N}$. Тогда

1) $A$ әндотранзитивна тогда и только тогда, когда $A=\bigoplus_{i=1}^{n} A_{i}$, где $A_{i}-$ квазиоднородные әндотранзитивные группь из $\mathscr{L} \cap \mathcal{N}$ или әндотранзитивные группы из следствия $6, u \Pi\left(A_{i}\right) \cap \Pi\left(A_{j}\right)=\varnothing$ при $i \neq j(i, j=1, \ldots, n)$;

2) эндотранзитивность $A$ влечет ее транзитивность, причем если все $A_{i}$ из 1) однородны, то $A$ вполне транзитивна.

ДокАЗАТЕЛЬСтво. 1) Необходимость. Согласно лемме $1 \alpha A(t) \neq 0$ для некоторого максимального типа $t$. Если $\operatorname{Ker} \alpha$ максимально, то по теореме $4 A=B \oplus D$, где $D \in \mathscr{N}$. Так же, как в следствии $6, B$ удовлетворяет посылке следствия 7. Поэтому применяя теорему 4 несколько раз, получим, что $A=\bigoplus_{k=1}^{m} D_{k}$, где $D_{k} \in \mathcal{N}$. Заметим, что если $\Pi\left(D_{k}\right) \cap \Pi\left(D_{s}\right) \neq \varnothing$ при $k \neq s$, то согласно лемме $3 D_{k}$ и $D_{s}$ - однородные групшы, содержащие ненулевые элементы максимальньх типов, поэтому типы этих групп одинаковы. Отсюда вытекает существование указанных прямых слагаемых $A_{i}$.

Достаточность. Нетрудно показать, что если $A=\bigoplus_{i=1}^{n} A_{i}$ и $\Pi\left(A_{i}\right) \cap \Pi\left(A_{j}\right)=\varnothing$ при $i \neq j$, то $A$ эндотранзитивна тогда и только тогда, когда все $A_{i}(i \in I)$ - эндотранзитивные группы $[1$, предложение 1.3$]$. Далее, всякая эндотранзитивная групша $D \in \mathscr{N}$ транзитивна. Группа $A=\bigoplus A_{i}$, где $A_{i}$ - изоморфные однородные вполне транзитивные группы из класса $\mathscr{N}$, обладает таким свойством, что любой ее элемент можно вложить 
в прямое слагаемое группы $A$, изоморфное $A_{i}[1$, лемма 3.3$]$, откуда следует транзитивность $A$. В частности, из сказанного вытекает справедливость 2).

ЗАмЕчАниЕ. Существуют транзитивные, но не вполне транзитивные группы из $\mathscr{N}$ даже конечного ранга [2, пример 7.2]. Эндотранзитивные группы из $\mathscr{L} \cap \mathscr{N}$ описаны в [1]. Поэтому следствие 7 полностью описывает рассматриваемые в нем группы. Любые прямые разложения эндотранзитивной групшы из следствия 7 в прямую сумму групा из класса $\mathscr{N}$ изоморфны; это доказьвается аналогично следствию 4.5 из [1].

\section{СПИСОК ЦИТИРОВАННОЙ ЛИТЕРАТУРЫ}

[1] Добрусин Ю. Б. О продолжениях частичных эндоморфизмов абелевых групп без кручения // Абелевы группы и модули. Вып. 4. Томск, 1986. С. 36-53.

[2] Добрусин Ю. Б. О продолжениях частичных эндоморфизмов абелевых групп без кручения, 2 // Абелевы группы и модули. Вьп. 5. Томск, 1985. С. 31-41.

[3] Крылов П.А. Сильно однородные абелевы группы без кручения // Сиб. матем. ж. 1983. T. 24. № 2. C. 77-84.

[4] Крылов П. А. Вполне транзитивные абелевы группы без кручения // Алгебра и логика. 1990. T. 29. № 5. C. 549-560.

[5] Чехлов А. Р. Связные квазисервантно инъективные абелевы группы // Изв. вузов. Матем. 1989. № 10. C. $84-87$.

[6] Arnold D. M. Strongly homogeneous torsion-free abelian groups of finite rank // Proc. Amer. Math. Soc. 1976. V. 56. P. 67-72.

[7] Dugas M., Shelah S. E-transitive groups in L // Contemp. Math. 1989. V. 87. P. 191-199.

[8] Hausen J. E-transitive torsion-free abelian groups // J. Algebra. 1987. V. 107. № 1. P. 17-27.

[9] Vinsonhaler C., Wickless W. A generalization of quasi-pure injective torsion-free abelian groups of finite rank // Houston J. Math. 1996. V. 22. № 3. P. 473-484.

[10] Беккер И.Х.,Крылов П. А., Чехлов А.Р. Абелевы группы без кручения, близкие к алгебраически компактным // Абелевы группы и модули. Вып. 11, 12. Томск, 1994. С. 3-52.

[11] Михалев А. В., Мишина А. П. Бесконечные абелевы группы: методы и результаты // Фундамент. и прикл. матем. 1995. Т. 1. № 2. С. 319-375. 